\title{
Application of Synthetic Biology to Regenerative Medicine
}

Elise Cachat and Jamie A. Davies*

Centre for Integrative Physiology, University of Edinburgh, Hugh Robson Building, George Square, Edinburgh EH8 9XD, UK

\begin{abstract}
Synthetic biology uses interchangeable and standardized "bio-parts" to construct complex genetic networks that include sensing, information processing and effector modules: these allow robust and tunable transgene expression in response to a change in signal input. The rise of this field has coincided closely with the emergence of regenerative medicine as a distinct discipline. Unlike synthetic biology, regenerative medicine uses the natural abilities of cells to make trophic factors and to produce new tissues as they would in normal development and tissue maintenance. In this article, we argue that bringing these young fields together, so that synthetic biology techniques are applied to the problem of regeneration, has the potential significantly to enhance our ability to help those in clinical need. We first review the synthetic tool kit available for engineered mammalian networks, then examine the main areas in which synthetic biology techniques might be applied to promote regeneration: (i) biosynthesis and controlled release of therapeutic molecules, (ii) synthesis of scaffold material, (iii) regulation of stem cells, and (iv) programming cells to organize themselves into novel tissues. We finally consider the long-term potential of synthetic biology for regenerative medicine, and the risks and challenges ahead.
\end{abstract}

Keywords: Synthetic biology; Regenerative medicine; Tissue engineering; Mammalian cells; Synthetic gene networks; Genetic engineering

\section{Introduction}

Synthetic biology has been defined, in a report by the Royal Academy of Engineering, as a discipline that aims to design and engineer biologically based parts, devices and systems [1]. It is a new but very rapidly growing field that has attracted considerable attention from researchers, governments and the public for its potential to change, quite profoundly, our relationship with the living world and our power to manipulate it [2-9]. The rise of this field has coincided closely with the emergence of regenerative medicine as a distinct discipline (Figure 1), both taking off rapidly in the early years of this century. In this article we argue that bringing these two young fields together, so that synthetic biology solutions are applied to problems of regeneration, has the potential to significantly enhance our ability to help those in clinical need.

Traditional efforts in regenerative medicine have always depended on using the natural, evolved behaviours of human cells. The earliest to be exploited were those concerned with wound healing and with tissue engraftment: together, these form the basis of reconstructive surgery, whether this is routine skin grafting or highly experimental transplantations of a hand or even a face (reviewed by [10]). From the middle of the last century, first researchers and then clinicians began to exploit an additional feature of the normal body, the ability of stem cells to regenerate missing tissue. Use of stem cells from bone marrow to reconstitute the haematopoietic system was achieved in the $1950 \mathrm{~s}$ in both rodents [11] and humans [12]: it has since become a relatively routine procedure following treatment for leukaemia or following accidental exposure to ionizing radiation. This century, the range of stem cell treatments has expanded greatly, at least in the experimental phase, and includes treatment with a variety of stem cells (including mesenchymal, perivascular, endothelial, neuronal, embryonic and limbal) with the aim of treating diseases such as diabetes, paraplegia, ataxia, multiple sclerosis, and heart failure (reviewed in [13]). So far, the clinical outcomes of most early trials have been disappointing, although the increasing commitment of industry to exploring this field suggests that optimism is far from lost. In all cases, though, the aim has been to use the natural abilities of cells to make trophic factors and to

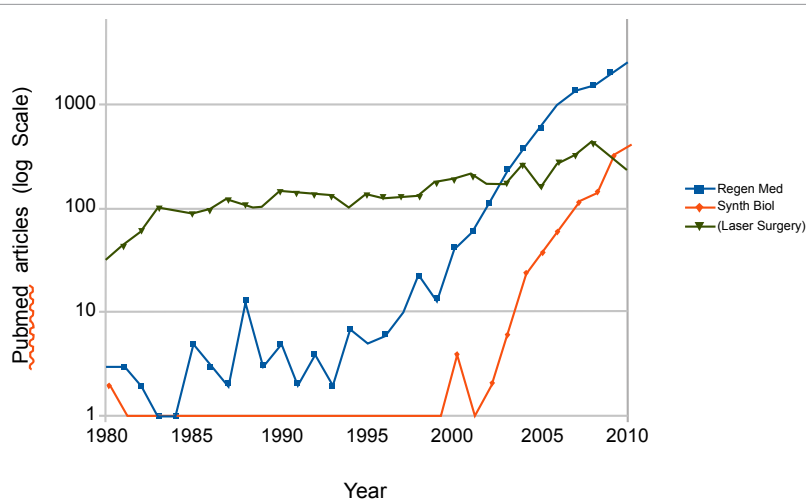

Figure 1: The rise of synthetic biology and regenerative medicine. Regenerative medicine is shown in blue and synthetic biology, taking off later but now with a similar trajectory, in red. The green line shows publications for laser surgery, chosen as a 'control' for the general gentle rise in medical publication. This graph was obtained by performing a PubMed search using the term XXXX NNNN, where XXXX was "synthetic biology", "regenerative medicine" or "laser surgery" and NNNN was each year in the range 1980-2010.

produce new tissues as they would in normal development and tissue maintenance.

An even more recent development has been the idea of engineering 'scaffolds', either wholly artificial or made by manipulation of natural molecules or mixtures of artificial and natural molecules, to guide cells to make tissues with specific shapes [14]. Examples include

*Corresponding author: Jamie A. Davies, Centre for Integrative Physiology University of Edinburgh, Hugh Robson Building, George Square, Edinburgh EH8 9XD, UK; Tel: 44-0-1316502999; Fax: 44-0-1316506545; E-mail: jamie.davies@ ed.ac.uk

Received November 18, 2011; Accepted December 05, 2011; Published December 09, 2011

Citation: Cachat E, Davies JA (2011) Application of Synthetic Biology to Regenerative Medicine. J Bioengineer \& Biomedical Sci S2:003. doi:10.4172/2155 9538.S2-003

Copyright: $\odot 2011$ Cachat E, et al. This is an open-access article distributed unde the terms of the Creative Commons Attribution License, which permits unrestricted use, distribution, and reproduction in any medium, provided the original author and source are credited. 
the formation of a collagen pattern in the shape of a human nipple [15], the formation of functional heart valves [16], reconstruction of cartilage [17], bone [18], oesophagus [19] and bladder wall [20]. In a variation on that theme, the engineering of structures that already incorporate cells in situ has been used to produce functional heart tissue [21]. Natural, de-cellularized scaffolds obtained by detergentwashing matrices of natural organs have also been used successfully for tissue engineering [22]. The possibility of engineering a matrix that has a shape different from that of any natural tissue (for example, for reconstruction of a body altered by accident or congenital abnormality) allows some creativity, but the actions of the cells themselves are still controlled by their evolved 'developmental programmes'. This fact imposes a significant limitation on the scope of conventional tissue engineering. It is in liberating the field from this limitation that the potential of synthetic biology lies.

Synthetic biology differs from traditional 'genetic engineering' in several ways. First, it tends to involve not just the manipulation of one gene but the construction of complex, larger-scale genetic systems. Second, most of its practitioners stress an 'engineering approach' using standard components and modules as $m$ ble: effectively a move from a 'craft' phase towards an 'industrial' phase of biological engineering [23]. With the design and construction of complex modular systems from well-characterized parts, a wide range of new applications becomes available, as illustrated by the use of new terms such as 'synthetic tissue biology' [24], 'synthetic morphology' [25] and 'synthetic toxicology' [26]. As in other fields, the potential inputs of synthetic biology to regenerative medicine can be of two kinds: the first aiding understanding and the second directly aiding a patient. Through de novo design, synthetic biology can help elucidate cellular mechanisms and pathways: engineering entirely or partially synthetic genetic networks in living cells gives us clues as to how natural systems are assembled and how they operate in organisms [27]. Through rational design based on this knowledge, synthetic biology has the potential to create new systems for diagnosis or treatment. In particular, it has enormous potential for tissue engineering and regenerative medicine in general.

In this review, we will provide an overview of some possible applications of synthetic biology to regenerative medicine. We will detail the synthetic tool kit already available for engineered mammalian networks, then review the first, tentative steps that have been made in applying synthetic biology to tissue engineering and to stem cell biology. We finally consider the long-term potential of synthetic biology for regenerative medicine, and the risks and challenges ahead.

\section{Areas for application of synthetic biology techniques}

There are four main areas in which the techniques of synthetic biology might be applied to promote regeneration;

- Biosynthesis and controlled release of therapeutic molecules

- Synthesis of scaffold material

- Regulation of stem cells

- Programming cells to organize themselves into novel tissues.

The rest of this chapter will first describe existing tools of synthetic biology that might be useful in all of these areas, and will then consider how they might be applied to each area in turn.

\section{Existing components for mammalian synthetic biology}

Most synthetic biology is based on the use of interchangeable and standardized 'bio-parts'. These are epitomized by the rapidly-growing collection of 'Bio-Bricks' made available to contestants in the annual iGEM competition [28], in which teams of undergraduates and even high school students compete to make 'the best' synthetic biological system. The parts are designed to be combined using standardized reactions, based mainly on restriction-ligation for prokaryotic systems and recombination-mediated cassette exchange for mammalian systems. Using these parts, complex genetic networks comprising sensing, information processing and effector modules can be (and have been) assembled through a systematic engineering approach not dissimilar, in principle, from the approaches used in the assembly of standard electronic components to make different circuits. The resulting synthetic systems allow robust and tunable transgene expression in response to changes in input signals. During the first wave of synthetic biology, around the turn of the century, bio-parts such as the toggle switch [29] and the oscillator [30] were developed in prokaryotes. Since 2004, the scope of synthetic biology has been extended to mammalian cells (reviewed in [31-33]). Below is a non-exhaustive list of significant mammalian parts illustrated by some applications.

\section{The synthetic tool kit}

Synthetic Boolean logic gates (AND, OR, NOR, etc.) have been engineered in mammalian cells [34] where they regulate transgene expression by transcriptional or post-transcriptional control. Those circuits respond to the presence or absence of different small molecule inputs (often antibiotics). They can be layered to yield more intricate functions, even though it remains difficult to predict the behaviour of complex combinations. Introducing feedback loops in such circuits allows the design of systems showing hysteretic signal integration [35] or bimodal switch characteristics [36]. Rinaudo et al. [37] have engineered a 'universal' RNAi-based Boolean logic evaluator in which the molecular endogenous inputs are encoded by small interfering RNAs (siRNAs). The system can make decisions with up to five logic variables and uses computer-designed siRNA molecules for minimal crosstalk within the network. This synthetic 'switchboard' could be engineered in cells to respond to different environmental and intracellular variables $(\mathrm{pH}$, light, metabolic state, insulin concentration, etc.) [38], making it a powerful tool for biomedical applications, both diagnostic and constructive.

Another logic circuit, the memory latch or toggle switch, was initially designed in E. coli [29] by combining two repressors, each of which controlled the other's expression. Kramer et al. [39] developed the PIPON/EON-based epigenetic toggle switch in Chinese hamster ovary cells where transient administration of one of two alternate antibiotics switched the system between two meta-stable transgene expression states. In other words, the cells 'remembered' their exposure to one drug until the memory was reset ('erased') by treatment with the other. These circuits are particularly useful to program mammalian processes with binary characteristics such as differentiation or apoptosis. This transcriptional switch was later improved and used in living mice [40], demonstrating its potential for precise long-term protein delivery in the absence of a sustained input, a critical feature when long term drug administration is potentially toxic or expensive.

Recently, Levskaya et al. [41] designed a light-inducible control system that can be used to sculpt mammalian cell shape. The circuit, based on an engineered, plant-derived light-sensitive protein and a reversible protein-protein interaction, recruits specific target proteins to the membrane. When the target protein in question is a modified activator of cytoskeletal organization, as it was in the paper, a beam of light can direct the formation of a lamellipodium. Such light-sensitive response elements could, in principle, be used in tissue engineering 
for the controlled activation of various cellular processes in a spatiotemporal manner. This is especially true in vitro, where access by light is easy, but may also be useful in some surface locations in vivo.

Tigges et al. [42] have adapted a synthetic oscillator design first demonstrated in bacteria [30] to develop robust and tuneable oscillations in mammalian cells. Coupling a positive feedback loop and a time-delayed negative feedback loop, this synthetic circuit showed autonomous and self-sustained oscillating gene expression. Another semi-synthetic oscillating genetic circuit features independent tuning of the amplitude, damping rate and oscillation frequency in human cells [43]. Such circuits provide insight into the complex mechanisms and perturbations that control dynamics in natural biological systems like circadian clocks. They can be implemented in systems where periodic synthesis and release of output molecules are needed and could provide sophisticated kinetic control for delivery of therapeutic agents in vivo.

Greber et al. [44] constructed a synthetic genetic network with band-pass detection characteristics; output expression is 'off' where there is no signal, 'on' across a window of low concentrations of input but 'off' again when the input concentration is high. Such a system is very interesting from the point of view of tissue engineering because it can be used for spatial patterning. If, for example, a signalling molecule is produced from one source and diffuses to create a gradient, the responsive gene will be 'on' in a halo around that source, demarcating a definite zone of tissue (Figure 2). If the responsive gene were itself a second, less diffusible, signalling molecule capable of driving another band-pass circuit (responsive only to this second molecule), a second layer of finer pattern would be added. Such systems could be combined to create highly complex predictable patterns. Another way of creating complex pattern would be coupling oscillator and bandpass to control transgene expression. The well-known 'somite clock' that divides paraxial mesoderm into a series of blocks (the pre-pattern for vertebrae) is effectively a coupling of a band-pass system with an oscillator, with the additional feature that, once a band of tissues has decided to respond, it becomes a new source for the signal.

Engineering cell-cell communication in E. coli, exploiting molecules used by various bacterial species for quorum sensing, has allowed multicellular quorum-sensing networks to detect population and to regulate it automatically by controlling cell death [45]. Basu et al. [46] also programmed multicellular populations to form patterns from an initially undifferentiated lawn, where 'receiver' cells differentiate on detection of a signal gradient synthesized by 'sender' cells. Building on prokaryote cell-cell communication designs, Weber et al. [47] fused bacterial quorum-sensing receptors with eukaryotic elements and constructed a signalling network consisting of human sender cells and $\mathrm{CHO}$ receiver cells with $\mathrm{L}$-arginine as the signalling molecule. In principle, these signalling systems can operate in three-dimensions (3D), regulating cell density in the volume of an artificial tissue, so that the tissue can maintain itself in the face of attrition.

We expect this list of mammalian bio-parts to grow rapidly in the coming years as other elegant prokaryotic designs (genetic counter

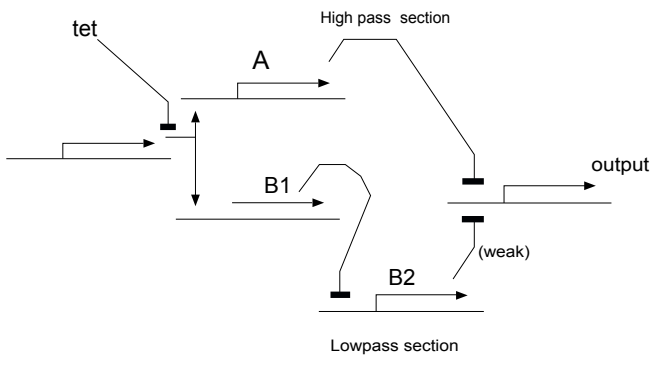

b

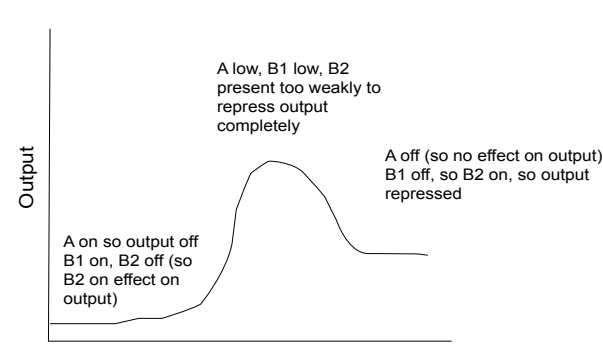

Tet concentration
C
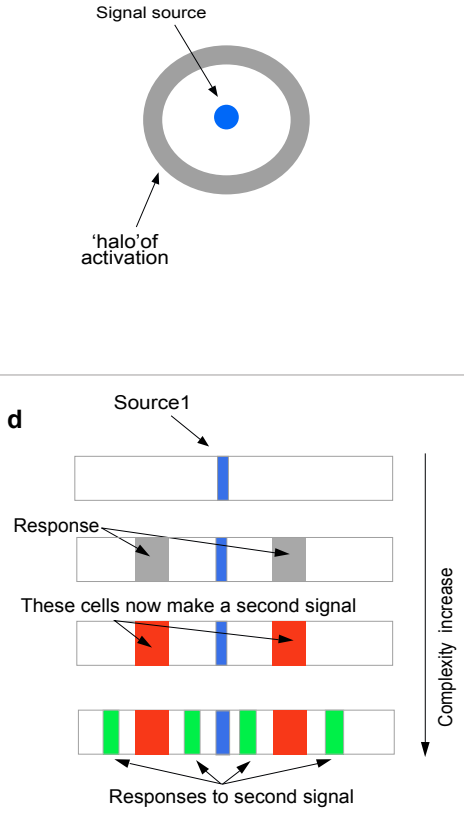

Figure 2: Synthetic band-pass filters as potential patterning elements. (a) depicts the architecture of the system built by Greber \& Fussenegger [44], which is based on two separate pathways that can each inhibit the production of the output molecule. The top pathway, on its own a high-pass filter, makes molecule A to repress the production of output molecule unless tetracycline (tet) is present at high enough concentrations to inhibit production of A. The bottom pathway, a low-pass filter, includes an extra inverting step so that in the absence of tet, B1 is made and represses the production of B2 so there is no B2 to repress the output (the promoters of B2 and the output are 'on' unless repressed, the promoters of A and B1 are off unless activated). When tet is present, B1 is repressed and B2 is made. B2 is a less potent repressor of the output than is $\mathrm{A}$, so that there is a window of tet concentration that suppresses $\mathrm{A}$ enough to allow some production of output and represses $\mathrm{B} 1$ enough that some B2 is made, but not enough to completely suppress the output either. (b) The circuit therefore allows production of significant output over a narrow band of tet concentration. (c) If tet (or some other signalling molecule in an appropriately modified version of the circuit) were present in a gradient from one source, the band-pass filter would result in the production of the output in a halo around that source. (d) If the output were itself a signal that diffused less rapidly, and a second band-pass circuit responsive to this signal were present, another layer of detail would be added to the pattern. In this way, synthetic circuits could pattern tissues and, in principle, control location-dependent differentiation of cells. 
[48], edge detection program [49], synchronized genetic clock [50], etc.) will in all probability be adapted to the mammalian chassis.

It is important to note that, as in other areas of engineering, modules-of-modules are likely to become important building blocks in construction: for example, transistors are 'components' at a low level, while fixed arrangements of transistors (in an integrated circuit) can be considered 'basic components' at higher level, and fixed arrangements of integrated circuits (e.g. data servers) can be considered 'basic components' at another level still. The 'BioBricks' available so far are basic DNA parts (promoters, protein-coding domains, etc.) or combinations of parts (logic gates, signal senders, etc.). Weber et al. [47] have considered making larger-scale parts available on the BioBrick registry: the so-called 'CytoBricks' are well-characterized mammalian cell lines with stably integrated synthetic networks. Applying this building blocks concept to yet other scales, we may envisage engineering whole tissue parts [24] or even organ parts (intraor extra-corporeally).

\section{Application area 1: biosynthesis and controlled release of therapeutic molecules}

One of the most surprising results to emerge from studies of stem cell-assisted regeneration is that many grafted stem cells seem to assist the regeneration of damaged host tissues not by directly giving rise to new replacement parts but by secreting factors that induce endogenous cells to repair the tissue [51,52]. Given concerns about the safety of introducing multi- and pluripotent cells into a human host (with attendant risk of tumour formation [53]), there is much interest in identifying the factors involved and applying them without transferring stem cells as well. There is a problem, though, that these molecules are likely to be proteins that will not be absorbed intact if given orally, and long-term treatments will be required.

One way of circumventing these problems in delivering largemolecule, regeneration-promoting therapeutics such as proteins to the inside of the body is to have them synthesized by cells already inside, at the right place. The rate of this synthesis can then be controlled either by medically-applied small molecules (drugs that pass easily through the gut or skin) or, in more advanced designs, by feedback loops designed into the synthetic system.

The idea of controlling a synthetic circuit embedded into the body by externally-applied small molecules has been demonstrated in a proof-of-principle experiment by Gitzinger et al. [54], who constructed a transdermally-controllable transcription system in mammalian cells using the apple-derived metabolite phloretin as a non-toxic trigger. This synthetic, phloretin-adjustable control element was able to adjust target gene expression reversibly in transgenic cells that had been implanted into mice, with the phloretin applied as a skin lotion. Another example of control by small molecules is the vitamin $\mathrm{H}$ (biotin)-triggered genetic switch [55].

Light can also be used to control transgene expression in mammalian cells. Ye et al. [56] created a synthetic light-inducible transgene expression device by connecting the photopigment melanopsin to the Nuclear Factor of Activated T cells (NFAT) pathway for controlling specific gene expression. In their engineered cells, illumination caused the expression of a variant of the human glycoprotein secreted alkaline phosphatase that displays potent glucose modulating characteristics. When the device was implanted transdermally in mice, transgene expression was regulated through direct illumination and was able to attenuate glycaemic excursions in type II diabetes.
The ability of synthetic biologists to engineer systems that feature closed-loop feedback control creates the potential for homeostasis to control the concentration of a target molecule, be it a drug or, even more usefully, a clinically-relevant outcome. A vivid illustration of this approach has been provided by Kemmer et al. [57], who constructed a synthetic system to control blood urate levels in animals that could not do this for themselves. Elevated urate causes the painful human condition, gout. The synthetic network, constructed in mouse cells, monitors urate levels in the blood and uses this signal to control expression of urate oxidase, an enzyme that degrades urate (Figure 3). The synthetic circuit was tested in urate oxidase-deficient mice and was shown to be effective.

None of these demonstrations address regenerative medicine directly, but they do illustrate the potential of the synthetic approach. Consider, for example, a variant of the urate system that, instead of controlling urate oxidase in response to fluctuating blood urate levels, synthesized insulin in response to fluctuating blood glucose. If it worked well enough, such a synthetic system (which could in principle be placed in any cell type, not just pancreatic $\beta$ cells) could make regeneration of a pancreas damaged by type I diab etes unnecessary. In this type of application, synthetic biology would be an alternative to, rather than a means of, tissue regeneration.

\section{Application area 2: production of improved scaffolds for tissue engineering}

Cell behaviour and shape are influenced strongly by the constitution and morphology of the extracellular matrix (ECM). Traditional tissue engineering has therefore included much effort in the production of carefully-engineered matrices, or 'scaffolds', to guide the growth and behaviour of engrafted cells to make a tissue. Synthetic 3D extracellular microenvironments that mimic the structure and functions of the ECM have already been developed [58], but synthetic biology holds tremendous potential for improving these matrices and making them structurally programmable.

Self-assembling peptide matrices form scaffolds that resemble cyto-architectures of tissues [59]. As in other fields of synthetic biology, these scaffolds are assemblies of known elements that can be considered building blocks [60]. They can incorporate different functional motifs to suit particular applications. Their molecular structures

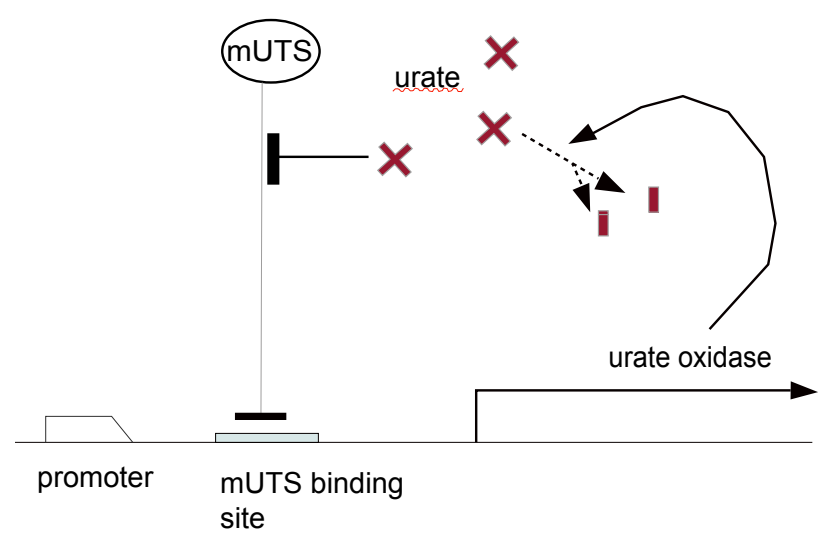

Figure 3: A synthetic circuit built by Kemmer et al. [57], that uses negative feedback to control the concentration of free urate. When no urate is present, the repressor mUTS efficiently suppresses the transcription of urate oxidase. When urate is present, it inhibits mUTS, allowing transcription of urate oxidase to proceed. The enzyme oxidizes (removes) urate. The system as a whole therefore works as a homeostat, keeping urate to low levels. 
are repeats of alternative hydrophilic and hydrophobic amino acids that spontaneously form $\beta$-sheets. Due to their intermolecular ionic interactions, those $\beta$-sheets can in turn be packed together under physiological conditions in a chequer-board-like fashion to form regular, high-density matrices where cells can be grown homogenously. Unlike common, natural biomaterials, the peptide scaffolds are pure and do not elicit inflammatory reactions in animals [61,62].

Hydrogels are flexible polymer matrices with a high water content: they can be engineered to include synthetic biology-derived parts to form interactive biohybrid materials for cell growth and tissue regeneration. Biological building blocks derived from proteins and DNA can be used for the construction of 'smart' hydrogels that are responsive to different stimuli. Those hydrogel structures have been extensively reviewed elsewhere ([63] for review) and we will give here only a brief overview.

DNA-based hydrogels can be engineered by crosslinking DNA to synthetic polymers such as polyacrylamide so that DNA molecules form bonds between branches of polymer. If complementary ssDNA oligonucleotides are applied to the gel, they spontaneously hybridize to the crosslinks and change the overall conformation of the gel. This can alter the gel's stiffness [64], volume [65,66], or induce its dissolution [67].

A combination of drug-inducible, protein-releasing synthetic biological modules in cells and synthetic hydrogels can be used to achieve dissolution on command. Ehrbar et al. [68,69] engineered a smart hydrogel where increasing concentrations of antibiotic resulted in the dissolution of the gel and the release of an embedded growth factor in a time- and dose-dependent manner (Figure 4). Such controlled dissolution is valuable for tissue engineering, allowing an artificial scaffold to be replaced, over time, with a new natural one [70]. Hydrogels that respond to physiological stimuli have also been constructed by incorporating engineered biological receptors that undergo reversible conformational changes on binding substrates such as calcium ions [71], glucose [72] or ATP [73].

Interestingly, Christen et al. [74] designed a stimulus-responsive hydrogel based on the conformational rearrangement of a transcription factor. This is based on the TetR/TetO system that is commonly used for inducible gene-expression systems. Again, the effect of the inducing drug was to cause dissolution of the gel. In principle, other protein/ DNA operator combinations could respond specifically to very diverse compounds such as drugs, metabolites, pheromones, etc. If similar modules are combined within the same gel, the resulting system could deal with several inputs and reacts accordingly through multiple mechanical and structural outputs.

The development of smart hydrogels such as the ones described above is of great potential importance to regenerative medicine where they could be used as scaffolds, tissue barriers, drug-delivery agents, to encapsulate cells or to recruit a different subset of cells [75]. Another interesting feature of those hydrogels is their spontaneous assembly: no enzyme is required as the molecular information for assembly is encoded in the 3D structure of the peptides. These ECM substitutes can be injected and hydrogelation triggered subsequently in vivo [60].

There are, as yet, very few demonstrations of clinically-relevant tissue engineering that includes a synthetic biological component. One

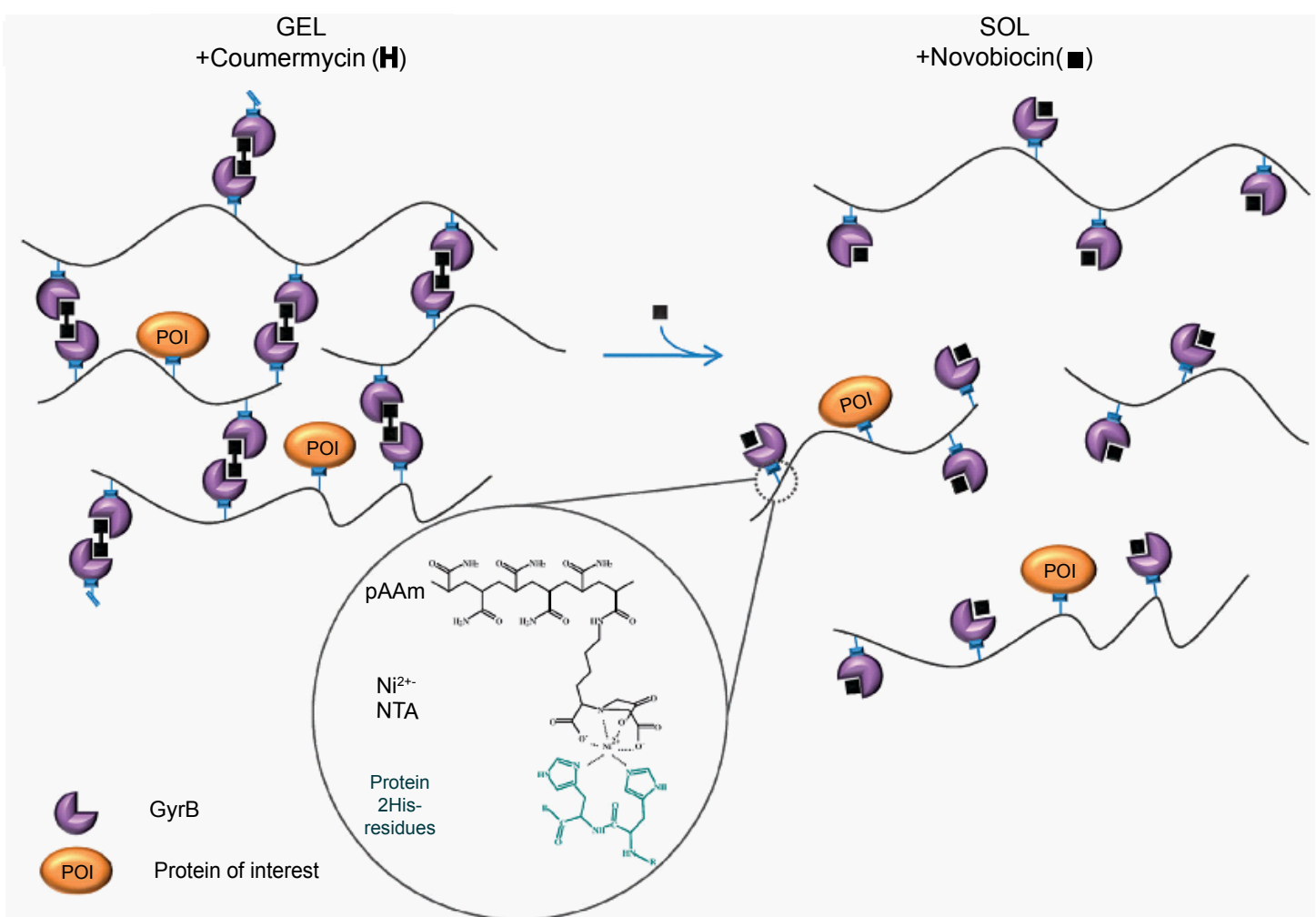

Figure 4: Drug-sensing GyrB-based hydrogel for the inducible release of biopharmaceuticals. The gyrase subunit $B$ (GyrB) was fused to a hexahistidine tag and dimerized by the antibiotic coumermycin. Mixing this protein with polyacrylamide (pAAm) functionalized with Ni2+-chelating nitrilotriacetic acid (NTA) resulted in the spontaneous formation of a hydrogel. Addition of novobiocin competitively prevented GyrB dimerization and triggered the dissolution of the hydrogel. This dissolution could be applied to trigger the release of a protein of interest (POI). Jakobus K, et al. [63] Synthetic mammalian gene networks as a blueprint for the design of interactive biohybrid materials. Chem Soc Rev, 2012 Reproduced by permission of The Royal Society of Chemistry. 
of the most spectacular was the combination of a cell encapsulation device with a synthetic biological circuit designed to achieve its controlled destruction. The application is in the field of animal fertility, specifically the insemination of cattle. As with natural mating, the success of artificial insemination depends on sperm being released into the female reproductive tract exactly when an egg has just been released into it. Kemmer et al. [76] encapsulated sperm in a cellulose-based capsule along with cells engineered to contain a hormone receptor that drove the synthesis of a cellulase. The hormone pulse accompanying ovulation caused synthesis of the cellulase, digestion of the cellulose capsule and consequent release of sperm onto the reproductive tract at just the right time.

\section{Application area 3: regulation of stem cells}

The area of regenerative medicine that is currently attracting most attention, from both scientists and public, is the construction of new tissue from stem cells. There are two ways in which synthetic biology could assist this effort even in the short term (more ways will probably be suggested as research progresses). These are (a) control of wild-type stem cells by a niche engineered using synthetic biological techniques, and (b) direct use of synthetic biology for stem cell 'programming'.

The niche of a stem cell - that is, the environment provided by neighbouring cells, their secreted ligands and the extracellular matrix - is a very important regulator of stem cell behaviour [77]. In particular, being completely in the niche or just out of it can determine whether a cell just formed by stem cell division remains in selfrenewal or commits to differentiation [78]. The techniques for scaffold construction outlined in the last section can be used to construct synthetic environments favourable to stem cell growth, where lack of resemblance to natural niches is compensated by a better control over the different components and an ability to switch the niche 'off' (i.e. to promote differentiation only) on command [79]. Smart hydrogels can also facilitate stem cell encapsulation [80], 3D-culture morphology [81] and stem cell embedding [82] for in vivo use.

The 'reprogramming' of differentiated cells to become pluripotent again is a very important area of stem cell research, because it allows the use of isogenic cells with those of a patient in need and it avoids ethically-contentious use of human embryos. We note in passing that 'reprogramming' is a very badly chosen word in this context, since the whole point is that the stem cells are shifted to a different (earlier) state in the natural human developmental programme. This state corresponds to that of the epiblast where cells can follow the human developmental programme a second time, according to the signals they now perceive. The phrase 'reprogramming', which fails to make the distinction between a 'programme' and a 'state' (a distinction made very clearly by Alan Turing at the dawn of computing in 1937), has now been broadly used and is sure to cause significant confusion in the era of synthetic biology, when reprogramming really will be performed.

There are two problems associated with induced pluripotential stem cells (iPSC): producing them in the first place and then controlling their differentiation. Since their discovery [83], iPSCs have been derived from adult cells with very low efficiency, by forcing the expression of four transcription factors (the 'KMOS set'): KLF4, c-MYC, OCT4 and SOX2. Better control of the process is urgently needed and synthetic biology may make its first contribution simply as a research tool. For example, synthetic circuits could be used to provide a very fine temporal regulation of KMOS expression to allow researchers to analyze the optimal pattern for more reliable iPSC production [38]. Once iPSC production has been optimized, synthetic modules may be of great value in driving whole cascades of sequential gene expression to control cell fate with increased fidelity [84].

\section{Application area 4: programming cells to construct designer tissues}

One approach that could avoid the use of stem cells altogether would be to program cells directly to construct 'designer tissues'. This would be a difficult and complex task but has the advantage that the tissues produced, either in vitro for use in extracorporeal life support machines or for transplantation to the body, or directly in situ, could be in arrangements that evolution has never produced.

A few years ago, one of the current authors published an outline of how this might be attempted [25]. The morphogenesis of natural embryonic tissues relies for the most part on about ten basic cell behaviours: proliferation, death, fusion, polarization, locomotion, chemotaxis, cell-cell adhesion, epithelial-to-mesenchymal transition, mesenchymal-to-epithelial transition and epithelial folding [85]. Each of these can be induced by the expression of a small number of regulatory proteins (often just one, given that the basic machinery is already available in a cell, waiting to be activated) [25]. It should therefore be possible to use control elements described in the overview of synthetic components near the beginning of this review to control the expression of these proteins and thereby to achieve 'programmable morphogenesis'. The authors are currently working on precisely this project, as is a lab in the USA. The nature of the work means that clinically-relevant applications will be many years away but the flexibility and power of the approach may make it ultimately one of the most medically-significant applications of synthetic biological techniques.

\section{Perspectives, challenges and dangers}

Technical challenges: With recent advances in synthetic biology and the emergence of applied mammalian circuits as exemplified earlier, there is a pressing need for the expansion of the synthetic mammalian bio-parts toolbox, as well as continuing the increase in the level of complexity and sophistication of engineered networks. Fully autonomous systems with tunable and predictable behaviour are required if they are ever to be used in vivo, where they must be able to evolve or adapt to environmental conditions. With more complex systems come more technical issues: crosstalk between modules and with host network, cell death, mutations, time delay in gene expression, noise, etc.

Another problem to overcome is the integration of large complex networks in mammalian cell genomes. With technological advances in gene synthesis, the limiting technology is no longer the construction of complex circuits but their integration in the cell. There is little control in mammalian cells as to where transgenes are integrated and genomic location can greatly influence their expression through transcriptional perturbations, chromatin remodelling or DNA methylation. Thus, new technologies are needed for stable and reproducible integration: tools such as zinc-finger nucleases (used for site-specific integration) [86] or insulator elements (used as shields from location effects). Ideally, synthetic biologists should develop tools for repairing damaged elements of synthetic networks or returning systems to their initial structure after unexpected modifications.

As the complexity of synthetic systems increases, inputs from systems biology and computational biology are required to (i) identify new components from endogenous networks; (ii) predict systems behaviour by modelling and fine-tuning through in silico runs before 
implementation; (iii) predict 3D development of structures and tissues; and (iv) visualize experimental 3D patterns.

A great number of technical challenges still need to be addressed in the field of tissue engineering. Complex features such as vasculature will need to be incorporated in engineered tissues where capillary networks are required for the exchange of nutrients and waste products. Ideally, engineered tissues should have regenerative powers to replace or repair damaged cells: stem cells, as well as elements of a healing response, should be incorporated. We are confident that synthetic biology can answer some of these issues, for example through the controlled spatiotemporal delivery of molecules such as growth factors for angiogenesis or anti-inflammatory cytokines for improved healing. Synthetic biologists might also need to engineer cells that can detect host/ synthetic boundaries for the regulation of host cell infiltration into the graft (and vice versa). To do so, systems allowing recognition and regulation of the 'synthetic-self' are needed. Other systems could assist in the targeting and repair of injured or aberrant synthetic tissues, where synthetic cells could detect their potential abnormal state. A recent study [87] reported a synthetic regulatory circuit that senses expression levels of endogenous microRNAs and triggers apoptosis in engineered HeLa cells where expression levels matched a predetermined profile. This 'cell-type classifier' demonstrates the possibility of a programmed response to a complex cellular state and can assist in the control of post-implantation behaviour. A major concern that still needs to be addressed in both tissue engineering and synthetic biology is the durability of systems. It is important that engineered tissues last over years or decades in vivo and that engineered cells, if integrated in grafts, stay operational for as long a period.

Perspective and dangers: This article is being written at, or rather just before, the dawn of the clinical application of synthetic biology and it is therefore full of prospects and predictions but very light on cases of use. Readers may, aware of the great distance between the small synthetic biology 'toys' that have been constructed so far and the applications we have outlined here, raise a note of scepticism and accuse the authors of unjustified hyperbole. The charge is reasonable; none of us can predict the future and the twentieth century contained many examples of apparently promising technologies that went nowhere (the flying car, the personal jet-pack), or were avoided due to a late appreciation of dangers (for example, nuclear-powered trains and cars). It is notable however, that many other technologies also began with a decade or two of amateurish and academic experimentation and proofs of principle, but then underwent an explosive and world-changing growth. A mere sixty years separates the Wright Brothers from the age of jet travel for all. Just thirty years took personal computing from the bedrooms of geeky kids who soldered together their own machines to the alwaysconnected, computer-in-the-pocket world of today. The progress of synthetic biology in general is being driven strongly by the need to replace fossil fuels and rare earth catalysts with plant- and microbederived equivalents, and it is notable that developing countries seem to be most alert to these needs and so may move fastest. With the essential tools being made anyway, it seems to the authors very unlikely that synthetic biology will not become, during the next few decades, an essential feature of regenerative medicine.

New technologies can bring new dangers. The direct introduction of synthetic genetic modules into the human body carries obvious dangers that the modules may malfunction, for example by mutation or epigenetic modulation, and cause their cells to do something dangerous. This problem, similar to that faced by stem cell biologists, is more worrying than the familiar one of adverse drug reactions because the cells will be harder to remove. We expect, for this reason, the first applications of synthetic biology to be extracorporeal, and the first in situ examples to involve cells physically encapsulated in an easilyremoved container, "just in case". It will probably be many years before such massively-engineered cells are allowed to roam freely around the body in routine Western medicine.

The experience of the computer industry should alert biotechnologists to the possibility that they will have deal not just with accidental harm but also with individuals or organizations causing deliberate harm, for the processes of extortion, terrorism or simply adolescent showing-off. The use of conventional or synthetic virology to construct novel pathogens that infect humans will probably be restrained by the obvious danger that they may infect anyone including the perpetrator and his comrades. If, though, one group of people begins to contain a significant number of individuals who carry novel synthetic DNA sequences not present in the rest of humanity, and especially if this group of people happen to be mainly the wealthy and powerful members of the world's economically and militarilydominant countries, that constraint may no long apply: it may be possible to design biological 'malware' (a literal virus) that can attack only those people. It may be sensible to consider these risks now, before we have become too committed to any particular technology, and to design defensively. The use of exactly the same components to construct everything may be a weakness in the face of potential attacks, and biological engineering may need to include significant diversity (the same arguments apply to the application of synthetic biology in the agricultural and bio-energy sectors). It may also be sensible, for reasons of self-interest as well as normal humanitarian ethics, to make advances in clinical synthetic biology available world-wide and not just to the rich, for if every nation has people whose lives have been saved by this technology and who carry its modules inside them, there will be no "us and them" to support the logic of an asymmetric attack.

These issues, and other more nebulous ones about human identity in an age of biological engineering, demand that scientists involved engage properly with ethicists, philosophers and the public. A failure to do so over the question of genetically-modified food resulted in a public backlash that has excluded the technology almost completely from the European Union. It would be a terrible mistake if we were to allow this to happen to synthetic biology too.

\section{Acknowledgements}

We would like to thank Kim Martin for her reading of this manuscript and for her suggestions.

\section{References}

1. Royal Academy of Engineering (2009) Synthetic Biology: scope, applications and implications.

2. Liang J, Luo Y, Zhao H (2011) Synthetic biology: putting synthesis into biology Wiley Interdisciplinary Reviews: Systems Biology and Medicine 3: 7-20.

3. Fritz BR, Timmerman LE, Daringer NM, Leonard JN, Jewett MC (2010) Biology by design: from top to bottom and back. J Biomed Biotechnol 2010: 232016.

4. Khalil AS, Collins JJ (2010) Synthetic biology: applications come of age. Nat Rev Genet 11: 367-379.

5. Dellomonaco C, Fava F, Gonzalez R (2010) The path to next generation biofuels: successes and challenges in the era of synthetic biology. Microb Cell Fact 9: 3.

6. Kelle, A (2009) Synthetic biology and biosecurity. From low levels of awareness to a comprehensive strategy. EMBO Rep 10 Suppl 1: S23-27.

7. Weber W, Fussenegger M (2009) The impact of synthetic biology on drug discovery. Drug Discovery Today 14: 956-963. 
Citation: Cachat E, Davies JA (2011) Application of Synthetic Biology to Regenerative Medicine. J Bioengineer \& Biomedical Sci S2:003. doi:10.4172/2155-9538.S2-003

8. Price GM, Chu KK, Truslow JG, Tang-Schomer MD, Golden AP, et al. (2008) Bonding of macromolecular hydrogels using perturbants. J Am Chem Soc 130: 6664-6665.

9. Benner SA, Sismour AM (2005) Synthetic biology. Nat Rev Genet 6: 533-543.

10. Gander B, Brown CS, Vasilic D, Furr A, Banis JC Jr, et al. (2006) Composite tissue allotransplantation of the hand and face: a new frontier in transplant and reconstructive surgery. Transplant International 19: 868-880.

11. Congdon CC, Uphoff D, Lorenz E (1952) Modification of acute irradiation injury in mice and guinea pigs by injection of bone marrow; a histopathologic study. $\mathrm{J}$ Natl Cancer Inst 13: 73-107.

12. Humble JG, Newton KA (1958) Technique of human bone-marrow transplants. Lancet 1: 142.

13. Trounson A, Thakar RG, Lomax G, Gibbons D (2011) Clinical trials for stem cell therapies. BMC Med 9: 52.

14. Prestwich GD (2008) Engineering a clinically-useful matrix for cell therapy Organogenesis 4: 42-47.

15. Cao YL, Lach E, Kim TH, Rodriguez A, Arevalo CA, et al. (1998) Tissueengineered nipple reconstruction. Plast Reconstr Surg 102: 2293-2298.

16. Shinoka T, Ma PX, Shum-Tim D, Breuer CK, Cusick RA, et al. (1996) Tissueengineered heart valves. Autologous valve leaflet replacement study in a lamb model. Circulation 94: II164-11168.

17. Kessler MW, Grande DA (2008) Tissue engineering and cartilage. Organogenesis 4: 28-32.

18. Davies JE, Matta R, Mendes VC, Perri de Carvalho PS (2010) Development, characterization and clinical use of a biodegradable composite scaffold for bone engineering in oro-maxillo-facial surgery. Organogenesis 6: 161-166.

19. Miki H, Ando N, Ozawa S, Sato M, Hayashi K, et al. (1999) An artificial esophagus constructed of cultured human esophageal epithelial cells, fibroblasts, polyglycolic acid mesh, and collagen. ASAIO J 45: 502-508.

20. Oberpenning F, Meng J, Yoo JJ, Atala A (1999) De novo reconstitution of a functional mammalian urinary bladder by tissue engineering. Nat Biotechno 17: $149-155$

21. Zimmermann WH, Melnychenko I, Wasmeier G, Didié $M$, Naito $H$, et al (2006) Engineered heart tissue grafts improve systolic and diastolic function in infarcted rat hearts. Nat Med 12: 452-458.

22. Baiguera S, Jungebluth $P$, Burns A, Mavilia C, Haag J, et al. (2010) Tissue engineered human tracheas for in vivo implantation. Biomaterials 31: 89318938.

23. Purnick PE, Weiss $R$ (2009) The second wave of synthetic biology: from modules to systems. Nat Rev Mol Cell Biol 10: 410-422.

24. Sia SK, Gillette BM, Yang GJ (2007) Synthetic tissue biology: Tissue engineering meets synthetic biology. Birth Defects Research Part C: Embryo Today: Reviews 81: 354-361.

25. Davies JA (2008) Synthetic morphology: prospects for engineered, selfconstructing anatomies. Journal of Anatomy 212: 707-719.

26. Schmidt M, Pei L (2011) Synthetic toxicology: where engineering meets biology and toxicology. Toxicol Sci 120 Suppl 1: S204-224.

27. Mukherji S, van Oudenaarden A (2009) Synthetic biology: understanding biological design from synthetic circuits. Nat Rev Genet 10: 859-871.

28. http://igem.org/Main_Page.

29. Gardner TS, Cantor CR, Collins JJ (2000) Construction of a genetic toggle switch in Escherichia coli. Nature 403: 339-342.

30. Elowitz MB, Leibler S (2000) A synthetic oscillatory network of transcriptional regulators. Nature 403: 335-338.

31. Weber W, Fussenegger M (2011) Molecular diversity--the toolbox for synthetic gene switches and networks. Curr Opin Chem Biol 15: 414-420.

32. Weber W, Fussenegger M (2010) Synthetic gene networks in mammalian cells Curr Opin Biotechnol 21: 690-696.

33. Aubel D, Fussenegger M (2010) Mammalian synthetic biology--from tools to therapies. Bioessays 32: 332-345.
34. Kramer BP, Fischer C, Fussenegger M (2004) BioLogic gates enable logical transcription control in mammalian cells. Biotechnol Bioeng 87: 478-484.

35. Kramer BP, Fussenegger M (2005) Hysteresis in a synthetic mammalian gene network. Proc Natl Acad Sci U S A 102: 9517-9522.

36. May T, Eccleston L, Herrmann S, Hauser H, Goncalves J, et al. (2008) Bimodal and hysteretic expression in mammalian cells from a synthetic gene circuit. PLoS One 3: e2372.

37. Rinaudo K, Bleris L, Maddamsetti R, Subramanian S, Weiss R, et al. (2007) A universal RNAi-based logic evaluator that operates in mammalian cells. $\mathrm{Nat}$ Biotechnol 25: 795-801.

38. Lu TK, Khalil AS, Collins JJ (2009) Next-generation synthetic gene networks Nat Biotech 27: 1139-1150.

39. Kramer BP, Viretta AU, Daoud-El-Baba M, Aubel D, Weber W, et al. (2004) An engineered epigenetic transgene switch in mammalian cells. Nat Biotechnol 22: $867-870$.

40. Greber D, El-Baba MD, Fussenegger M (2008) Intronically encoded siRNAs improve dynamic range of mammalian gene regulation systems and toggle switch. Nucleic Acids Res 36: e101.

41. Levskaya A, Weiner OD, Lim WA, Voigt CA (2009) Spatiotemporal control of cell signalling using a light-switchable protein interaction. Nature 461: 997-1001.

42. Tigges M, Marquez-Lago TT, Stelling J, Fussenegger M (2009) A tunable synthetic mammalian oscillator. Nature 457: 309-312.

43. Toettcher JE, Mock C, Batchelor E, Loewer A, Lahav G (2010) A synthetic-natural hybrid oscillator in human cells. Proc Natl Acad Sci U S A 107: 17047-17052.

44. Greber D, Fussenegger M (2010) An engineered mammalian band-pass network. Nucleic Acids Res 38: e174.

45. You L, Cox RS 3rd, Weiss R, Arnold FH (2004) Programmed population control by cell-cell communication and regulated killing. Nature 428: 868-871.

46. Basu S, Gerchman Y, Collins CH, Arnold FH, Weiss R (2005) A synthetic multicellular system for programmed pattern formation. Nature 434: 1130-1134.

47. Weber W, Schuetz M, Denervaud N, Fussenegger M (2009) A synthetic metabolitebased mammalian inter-cell signaling system. Mol Biosyst 5: 757-763.

48. Friedland AE, Lu TK, Wang X, Shi D, Church G, et al. (2009) Synthetic gene networks that count. Science 324: 1199-1202.

49. Tabor JJ, Salis HM, Simpson ZB, Chevalier AA, Levskaya A, et al. (2009) A synthetic genetic edge detection program. Cell 137: 1272-1281.

50. Danino T, Mondragon-Palomino O, Tsimring L, Hasty J (2010) A synchronized quorum of genetic clocks. Nature 463: 326-330.

51. Togel F, Westenfelder C (2011) The role of multipotent marrow stromal cells (MSCs) in tissue regeneration. Organogenesis 7: 96-100.

52. Harari-Steinberg O, Pleniceanu O, Dekel B (2011) Selecting the optimal cell for kidney regeneration: fetal, adult or reprogrammed stem cells. Organogenesis 7: 123-134.

53. Piscaglia AC (2008) Stem cells, a two-edged sword: risks and potentials of regenerative medicine. World J Gastroenterol 14: 4273-4279.

54. Gitzinger M, Kemmer C, Daoud-El Baba M, Weber W, Fussenegger M (2009) Controlling transgene expression in subcutaneous implants using a skin lotion containing the apple metabolite phloretin. Proceedings of the National Academy of Sciences 106: 10638-10643.

55. Weber W, Lienhart C, Daoud-El Baba M, Fussenegger M (2009) A biotintriggered genetic switch in mammalian cells and mice. Metab Eng 11: 117-124

56. Ye H, Daoud-El Baba M, Peng RW, Fussenegger M (2011) A synthetic optogenetic transcription device enhances blood-glucose homeostasis in mice. Science 332: 1565-1568

57. Kemmer C, Gitzinger M, Daoud-El Baba M, Djonov V, Stelling J, et al. (2010) Self-sufficient control of urate homeostasis in mice by a synthetic circuit. Nat Biotech 28: 355-360

58. Owen SC, Shoichet MS (2010) Design of three-dimensional biomimetic scaffolds. J Biomed Mater Res A 94: 1321-1331. 
Citation: Cachat E, Davies JA (2011) Application of Synthetic Biology to Regenerative Medicine. J Bioengineer \& Biomedical Sci S2:003. doi:10.4172/2155-9538.S2-003

59. Gelain F, Horii A, Zhang S (2007) Designer self-assembling peptide scaffolds for 3-d tissue cell cultures and regenerative medicine. Macromol Biosci 7: 544551.

60. Papapostolou D, Howorka S (2009) Engineering and exploiting protein assemblies in synthetic biology. Molecular BioSystems 5: 723-732.

61. Ellis-Behnke RG, Liang YX, You SW, Tay DK, Zhang S, et al. (2006) Nano neuro knitting: peptide nanofiber scaffold for brain repair and axon regeneration with functional return of vision. Proc Natl Acad Sci U S A 103: 5054-5059.

62. Davis ME, Hsieh PC, Takahashi T, Song Q, Zhang S, et al. (2006) Local myocardial insulin-like growth factor 1 (IGF-1) delivery with biotinylated peptide nanofibers improves cell therapy for myocardial infarction. Proc Natl Acad Sci U S A 103: 8155-8160.

63. Jakobus K, Wend S, Weber W (2012) Synthetic mammalian gene networks as a blueprint for the design of interactive biohybrid materials. Chem Soc Rev.

64. Lin DC, Yurke B, Langrana N.A. (2005) Inducing Reversible Stiffness Changes in DNA-crosslinked Gels. Journal of Materials Research 20: 1456-1464.

65. Tierney S, Stokke BT (2009) Development of an oligonucleotide functionalized hydrogel integrated on a high resolution interferometric readout platform as a label-free macromolecule sensing device. Biomacromolecules 10: 1619-1626.

66. Gao M, Gawel K, Stokke BT (2011). Toehold of dsDNA exchange affects the hydrogel swelling kinetics of a polymer-dsDNA hybrid hydrogel. Soft Matter 7: 1741-1746.

67. He X, Wei B, Mi Y (2010) Aptamer based reversible DNA induced hydrogel system for molecular recognition and separation. Chemical Communications 46: $6308-6310$

68. Ehrbar M, Schoenmakers R, Christen EH, Fussenegger M, Weber W (2008) Drug-sensing hydrogels for the inducible release of biopharmaceuticals. Nat Mater 7: 800-804.

69. Kämpf MM, Christen EH, Ehrbar M, Daoud-El Baba M, Charpin-El Hamri G et al. (2010) A Gene Therapy Technology-Based Biomaterial for the TriggerInducible Release of Biopharmaceuticals in Mice. Advanced Functional Materials 20: 2534-2538.

70. Rajagopal K, Schneider JP (2004) Self-assembling peptides and proteins for nanotechnological applications. Curr Opin Struct Biol 14: 480-486.

71. King WJ, Toepke MW, Murphy WL (2011) Facile formation of dynamic hydrogel microspheres for triggered growth factor delivery. Acta Biomater 7: 975-985.

72. Ehrick JD, Luckett MR, Khatwani S, Wei Y, Deo SK, et al. (2009) Glucose responsive hydrogel networks based on protein recognition. Macromol Biosci 9: 864-868.
73. Yuan W, Yang J, Kopeckova P, Kopecek J (2008) Smart hydrogels containing adenylate kinase: translating substrate recognition into macroscopic motion. $J$ Am Chem Soc 130: 15760-15761.

74. Karlsson M, Kämpf MM, Schoenmakers R, Gübeli RJ, et al. (2011) Conditiona DNA-Protein Interactions Confer Stimulus-Sensing Properties to Biohybrid Materials. Advanced Functional Materials 21: 2861-2867.

75. Slaughter BV, Khurshid SS, Fisher OZ, Khademhosseini A, Peppas NA (2009) Hydrogels in Regenerative Medicine. Advanced Materials 21: 3307-3329.

76. Kemmer C, Fluri DA, Witschi U, Passeraub A, Gutzwiller A, et al. (2011) A designer network coordinating bovine artificial insemination by ovulationtriggered release of implanted sperms. J Control Release 150: 23-29.

77. Ruiz SA, Chen CS (2008) Emergence of patterned stem cell differentiation within multicellular structures. Stem Cells 26: 2921-2927.

78. Kiefer JC (2011) Primer and interviews: The dynamic stem cell niche. Dev Dyn 240: 737-743.

79. Fisher OZ, Khademhosseini A, Langer R, Peppas NA (2009) Bioinspired Materials for Controlling Stem Cell Fate. Accounts of Chemical Research 43 : 419-428.

80. Anderson SB, Lin CC, Kuntzler DV, Anseth KS (2011) The performance of human mesenchymal stem cells encapsulated in cell-degradable polymerpeptide hydrogels. Biomaterials 32: 3564-3574.

81. Carpenedo RL, Bratt-Leal AM, Marklein RA, Seaman SA, Bowen NJ, et al. (2009) Homogeneous and organized differentiation within embryoid bodies induced by microsphere-mediated delivery of small molecules. Biomaterials 30: $2507-2515$.

82. Ballios BG, Cooke MJ, van der Kooy D, Shoichet MS (2010) A hydrogel-based stem cell delivery system to treat retinal degenerative diseases. Biomaterials 31: $2555-2564$

83. Takahashi K, Yamanaka S (2006) Induction of pluripotent stem cells from mouse embryonic and adult fibroblast cultures by defined factors. Cell 126 663-676.

84. Macarthur BD, Ma'ayan A, Lemischka IR (2009) Systems biology of stem cel fate and cellular reprogramming. Nat Rev Mol Cell Biol 10: 672-681.

85. Davies JA (2005) Mechanisms of Morphogenesis, Elsevier Academic Press

86. Cathomen T, Joung JK (2008) Zinc-finger nucleases: the next generation emerges. Mol Ther 16: 1200-1207.

87. Xie Z, Wroblewska L, Prochazka L, Weiss R, Benenson Y (2011) Multi-input RNAi-based logic circuit for identification of specific cancer cells. Science 333 1307-1311. 